\title{
Intervención breve en accidentados con alcoholemia positiva desde un centro de traumatología
}

\author{
Rodríguez-Martos, A. '; Plasència, A. ${ }^{2,4}$; Escayola, M. ${ }^{3}$; Martí, J. ${ }^{3}$; Ferrando, J. ${ }^{2}$; Torralba, Ll.' \\ (1) Plan de Acción sobre Drogas de Barcelona \\ (2) Instituto Municipal de la Salud: Instituto Municipal de Salut Pública, Ayuntamiento de Barcelona. \\ (3) Hospital de Traumatología y Rehabilitación: Hospitales Valle de Hebrón, Barcelona. \\ (4) Departamento de Medicina Preventiva y Salud Pública. Universidad Autónoma de Barcelona. \\ Enviar correspondencia a: \\ A. Rodríguez-Martos. Plan de Acción sobre Drogas de Barcelona. Plaza de Lesseps, 1. 08023 Barcelona.
}

\section{RESUMEN}

Objetivos: E1 objetivo principal de la investigación es comprobar la efectividad de la intervención breve para reducir el consumo de alcohol en accidentados de tráfico adultos con alcoholemia positiva y sin dependencia del alcohol.

En objetivo de la etapa piloto es comprobar la viabilidad estructural del proyecto (capacitación de los profesionales y viabilidad del cribado e intervención desde un centro de traumatología, así como una estimación aproximada de la prevalencia y perfil de los lesionados de tráfico con presencia de alcohol.

Material: Se elabora un programa de formación con material de apoyo. Se aplica un método de detección del alcohol en saliva en lesionados de tráfico que acuden a urgencias de traumatología. A los pacientes con saliva positiva se les ofrece una intervención breve. Se recoge la opinión del personal sobre la eficacia percibida y viabilidad estimada del proyecto gobal (cribado e intervención) mediante cuestionarios de opinión.. Se estudian la prevalencia y perfil de los pacientes con saliva positiva a partir del análisis comparativo de variables seleccionadas.

Resultados: El personal entrenado considera (80\%) útil y adecuada la formación recibida. Las enfermeras consideran (89\%) que el método es fácil, no plantea demasiados problemas $(55 \%)$ y es bien aceptado por el paciente (53\%). El personal entrenado se ha sentido cómodo y eficiente (67\%) al realizar la intervención. 261 de 264 pacientes aceptaron el cribado y el $11 \%$ tenía alcohol en saliva. Sólo 2 pacientes rechazaron la intervención, que fue practicada en 16 lesionados. Los pacientes con saliva positiva acuden más en fines de semana ( $p$ $0,005)$ y quedan ingresados con mayor frecuencia $(p 0,05)$ que aquéllos con saliva negativa.

Conclusión: el programa está bien estructurado, es viable y aceptado por el paciente. El estudio definitivo incluirá la determinación del alcohol en orina, en pacientes con saliva seca, y reforzará el cribado en fines de semana y politraumáticos.

Palabras clave: lesionados de tráfico, centro de traumatología, formación, cribado de alcohol, intervención breve.

\section{SUMMARY}

Objectives: Main research objective is to verify the effectiveness of brief intervention to reduce alcohol consumption in non-dependent adults injured in a traffic crash, presenting at the emergency department with a positive saliva alcohol test.

The aim of the pilot study is to verify the feasibility of the project (staff training, screening and intervention at a trauma centre) and to roughly estimate the prevalence and profile of alcohol-attributable traffic casualties.

Method: Training programme and support material are delivered. Saliva alcohol screening is performed by nurses in patients attending the emergency department because of a traffic crash. Positive patients are offered brief intervention. Staff's opinion on training, esteemed self-efficacy and feasibility of the whole procedure (screening and intervention) is collected by opinion surveys. Prevalence and profile of patients with positive saliva is drawn up by comparative analysis of selected variables.

Results: Trained staff considers (80\%) training useful and matching objectives. Nurses consider that, despite the nonvalid results in case of mouth dryness, the saliva test is easy (89\%), doesn't pose major problems (55\%), and is well accepted by the patient (53\%). Trained staff have felt comfortable and self-effective $(67 \%)$ in delivering the intervention. 261 out of 264 patients accepted screening and $28(11 \%)$ had a positive result. Only 2 patients refused intervention, which was performed on 16. Patients with a positive test present more frequently in the weekends (p: 0.005) and are more hospitalised (p: 0.05) than those with a negative test.

Conclusion: The programme is well designed, feasible and accepted by the patient. Main study will use urine alcohol test on patients with dry mouth, and will reinforce screening in the weekends and in sever injured patients.

Key words: traffic casualties, trauma centre, training, alcoholscreening, brief intervention. 


\section{INTRODUCCIÓN}

El objetivo principal del estudio que acometemos es comprobar la efectividad de la intervención breve para reducir el consumo de alcohol en accidentados de tráfico con alcoholemia positiva y sin dependencia del alcohol atendidos en un centro de traumatología.

Los criterios de inclusión en el estudio son: ser admitido en urgencias de traumatología por una lesión de tráfico, tener 18 o más años y presentar un test de alcohol en saliva positivo. El cribado de saliva se realiza en urgencias. La intervención se puede hacer en urgencias (en un momento post-agudo y antes del alta) o en sala (en las primeras semanas subsiguientes al accidente, en caso de hospitalización). La intervención no debe realizarse mientras el paciente esté clínicamente intoxicado o se encuentre en condiciones psicorgánicas críticas. Cuando se supone una dependencia alcohólica, la intervención se orienta a la derivación del paciente a tratamiento y el caso se excluye del análisis de efectividad.

La figura 1 resume el algoritmo de la intervención. Se presentan los resultados de la fase piloto, etapa ineludible del estudio definitivo.

\section{Objetivos de la fase piloto}

Objetivo principal: Comprobar la viabilidad del cribado de alcohol en saliva y de la intervención breve para la modificación del consumo de riesgo en los lesionados por accidente de tráfico con saliva positiva desde un centro de traumatología.
Objetivos operacionales:

- Comprobar la posibilidad de capacitar a los profesionales encargados de hacer la intervención

- Comprobar la viabilidad del cribado y de la intervención a partir de la opinión de los profesionales en cuanto a las dificultades percibidas en la ejecución del proyecto.

- Estimar la prevalencia y perfil de los lesionados de tráfico con presencia de alcohol durante el periodo consignado.

\section{Hipótesis}

La posibilidad de implantar un programa de Identificación Precoz e Intervención Breve en un centro de traumatología, y en un servicio de urgencias en particular, ha sido comprobada por diversos estudios, aunque con enfoque diverso: los servicios de urgencias se han concentrado más en la detección, y las clínicas traumatológicas, en la intervención.

En nuestro caso, el pequeño porcentaje de lesionados que ingresan nos ha obligado a intentar prestar la intervención también desde el entorno de urgencias, desde el que parece asumible a priori, contando con personal formado en la mayoría de turnos de enfermería. Se cuenta además con 2 asistentes sociales que podrian desplazarse, a demanda y en horario diurno, al servicio de urgencias o a la sala de hospitalización.

La literatura aporta ejemplos de la idoneidad del personal de enfermería ${ }^{13} y$, en menor medida, de trabajo social ${ }^{4}$ para realizar la identificación e intervención

Figura 1. Lesionados en accidente de tráfico con presencia de alcohol en saliva.

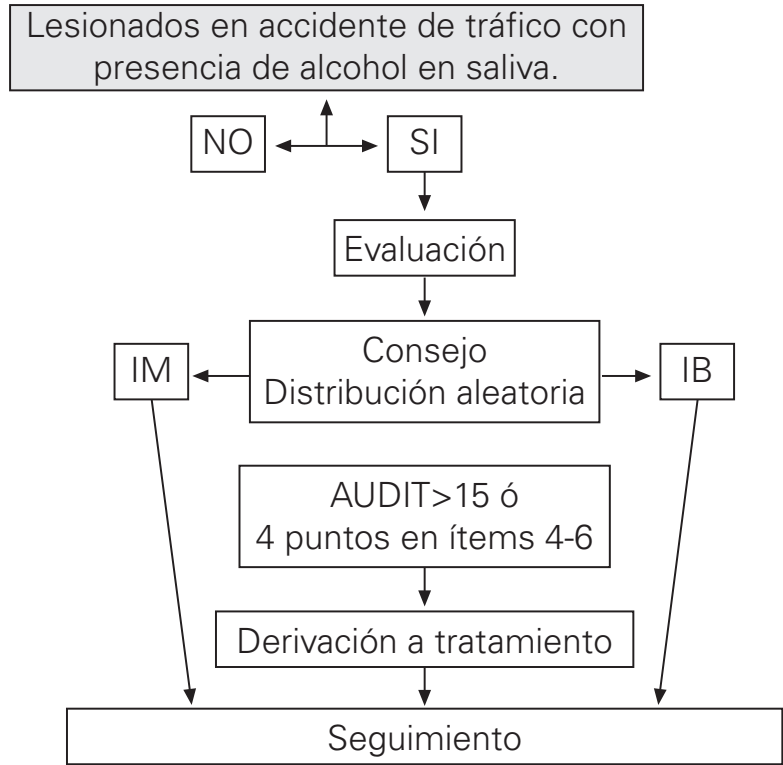


breve. Las experiencias suelen ser positivas, aunque más en el campo de la detección que de la intervención ${ }^{5}$. Los fallos que se informan ${ }^{6}$ son atribuidos a una falta de respaldo en la tarea encomendada y no parecen existir diferencias entre médicos y enfermeras no especializados ${ }^{7}$, sobre todo cuando reciben entrenamiento previo ${ }^{8}$. Aunque el entrenamiento referido en los estudios suele ser breve (1-2 horas), también se refiere la aplicación de seminarios. Su importancia ha sido repetidamente subrayada ${ }^{9}$, aportándose mejores resultados al unir entrenamiento grupal y supervisión individualizada ${ }^{10}$.

Se ha apuntado que el resultado de la intervención depende del estilo terapéutico y de la fidelidad al protocolo y tiempo empleado. ${ }^{11}$

Por lo que respecta a la viabiliad del cribado, la literatura indica que la determinación de alcohol en saliva es fácil, fiable, no invasiva e ideal para aplicar en un servicio de urgencias de traumatología ${ }^{12-14}$. A pesar de que muchas publicaciones avalan la concordancia entre los valores de alcohol en saliva y sangre ${ }^{15-19}$, estimada en general en torno a 1:1, a efectos legales la determinación de alcohol en saliva debe corroborarse con una determinación en sangre y no tiene valor en los tribunales, lo cual es una ventaja para los implicados en accidentes y para los servicios sanitarios.

Su uso en urgencias podría ser muy útil para dar una información rápida. Para obtener su máximo rendimiento es preciso conocer las ventajas y desventajas de cada método utilizado ${ }^{20}$.

La efectividad de la intervención breve viene avalada por abundante literatura ${ }^{21-22}$, en particular por lo que respecta a su uso en Atención Primaria. Su eficacia se ha atribuido a la incorporación de elementos cognitivoconductuales destinados a promover la motivación para el cambio. Su utilización en urgencias ${ }^{23-26}$ y en traumatología es objeto de creciente interés, al estimarse que un evento aversivo, como es el haber sufrido un accidente de tráfico, constituye un momento especialmente receptivo para un consejo de cambio. ${ }^{27-28}$ Diversos estudios aportan resultados alentadores ${ }^{29-32}$.

De acuerdo con la literatura, cabe esperar una prevalencia aproximada del $20-30 \%$ de casos con alcohol positivo en lesionados de tráfico ${ }^{33} \mathrm{y}$, dentro de este colectivo, una reincidencia 2,5 veces superior a los no intoxicados ${ }^{34}$. Una cuarta parte de los lesionados podría beneficiarse del programa previsto.

\section{MATERIAL Y MÉTODOS}

\section{Formació del personal}

Formar profesionales para abordar problemas de alcohol supone proporcionarles conocimientos y habi- lidades para intervenir sobre problemas de alcohol y fomentar en ellos el desarrollo de actitudes positivas con respecto a la adecuación de la intervención.

Profesionales de enfermería (8) y trabajo social (2) fueron reclutados en función del interés que manifestaron en participar (sondeo personal a cargo de la supervisora de enfermería), procurando que estuvieran representados la mayor parte de turnos de guardia.

Partiendo de las experiencias que aporta la literatura, así como de las propias propuestas para mejorar los programas de formación para la identificación precoz e intervención breve sobre problemas de alcohol en Europa ${ }^{35}$, se elaboró un programa de formación que incluía:

1) Entrega de un dossier con información básica especialmente elaborado para el proyecto, bibliografía, instrumentos diagnósticos a utilizar, guía de la intervención y material de soporte para la intervención, incluyendo dípticos para entregar al paciente.

2) Sesión docente interactiva (4 horas) con introducción de conocimientos básicos sobre el alcohol y los riesgos asociados a su consumo, con especial énfasis en sus consecuencias al volante; concepto y fundamento de la intervención breve, con introducción del modelo del cambio, el abordaje motivacional y los elementos activos de la intervención breve; introducción de los instrumentos de detección y presentación del modelo de intervención a implementar en el presente estudio, e introducción al role-playing.

3) Sesión práctica (4 horas) incluyendo: a) revisión del protocolo a utilizar, clarificando cualquier duda; b) utilización práctica del modelo mediante role-playing; c) discusión, dudas y sugerencias

4) Sesión de supervisión y refuerzo (2 horas), una vez iniciadas las intervenciones.

La formación se orientó a la capacitación de los agentes de intervención y tuvo en cuenta las disponibilidades horarias de todos los participantes, lo que implicó una flexibilidad absoluta y obligó a distribuir la formación en dos sesiones: matutina y nocturna, de unas 4 horas de duración. Aún así, alguna persona no pudo asistir íntegramente a las sesiones, lo que motivó que, en la sesión conjunta destinada a la supervisión y apoyo de las actividades, se incluyera un refuerzo de la formación práctica a demanda de los propios profesionales.

La opinión de los participantes sobre la formación recibida se recogió mediante encuesta autocontestada administrada una vez finalizada la formación teórico-práctica (sesiones 1 y 2) y antes de iniciar las 
intervenciones. Respondió la totalidad del personal entrenado $(\mathrm{N}=10)$.

\section{Detección de la presencia de alcohol en saliva}

Todo lesionado de tráfico de 18 o más años era candidato a la prueba.

Razones éticas nos llevaron a optar por un test de saliva cualitativo (Alcohol On-Site de Roche Diagnos$\left.\operatorname{tics}^{36}\right)$ que parecía ajustarse a nuestras necesidades. El método se basa en dos reacciones enzimáticas involucrando la alcohol- dehidrogenasa (ADH), el coenzima nicotinamida adenina dinucleótido (NAD) y una diaforasa. La muestra se recoge mediante dispositivo absorbente y desechable y se deposita en un contenedor de la bandejita de plástico suministrada, donde también se encuentra la cubeta en que previamente se ha colocado una gota de reactivo.

El test es cualitativo $y$, a concentraciones de $0,2 \mathrm{~g}$ $\mathrm{OH} / /$ de sangre, es positivo (color púrpura) a los 2 minutos (3-5 según Schwartz et al. ${ }^{21}$ ). La sensibilidad estimada es del $98 \%$ con alcoholemia 0,2 y del $100 \%$ con 0,25 de alcoholemia. La especificidad es prácticamente total, interfiriendo sólo el propanolol a una concentración de 0,05\% g/l, improbable de hallar en saliva humana (datos aportados por el fabricante).

El método fue presentado por la supervisora de enfermería a todos los turnos de urgencias. Las dificultades evidenciadas en la aplicación del método justificaron una ulterior demostración práctica, con entrenamiento en el manejo del reactivo para todos $y$ cada uno de los turnos.

La valoración que los profesionales hacían del cribado y la viabilidad estimada del mismo, se recogió mediante encuesta de opinión autocontestada a las 6 semanas de haber iniciado el cribado y cuando todos los participantes tenían experiencia personal en su manejo. Respondieron 20 personas.

\section{Intervención (evaluación y consejo)}

A todo paciente con saliva positiva se le ofrecía una intervención al tiempo que se le entregaba una carta de presentación del estudio. El primer paso consistía en evaluar la conducta y actitudes del paciente con respecto al alcohol y riesgos viarios asociados (Cuestionario AUDIT, Inventario de actitudes, conocimientos y conductas de riesgo vial, Escala de atribución del accidente al alcohol y Regleta del cambio). Esta evaluación comportaba unos 10'.

El diagnóstico CIE-9 y la Escala de Gravedad de la Lesión estaban previstos en la evaluación, pero deben ser aportados por el hospital a trimestre vencido.
La intervención mínima IM (5 minutos) incluía: 1) informar al enfermo de su situación: saliva positiva y nivel de riesgo detectado respecto a su consumo de alcohol; 2) explicación de los límites prudentes para el consumo de alcohol y de los riesgos del alcohol en la conducta viaria; 3) consejo de mantener el consumo dentro de los límites recomendables y no conducir cuando se bebe; 4) entrega de material informativo y de autoayuda.

La intervención breve IB (15-20 minutos) incluía, además de los apartados de la IM, una reflexión en torno a las "cosas buenas y malas" asociadas a la bebida para crear la disonancia cognitiva del paciente y promover su motivación para el cambio, aportaba sugerencias de conductas alternativas e incorporaba en conjunto los elementos activos conocidos bajo el acrónimo FRAMES.

Se elaboraron unas guías-modelo para la IM y para la IB, respectivamente. Se trata de guías concisas y fáciles de consultar en el curso de la intervención.

Junto con la guía, se facilitaba material de apoyo para la intervención (esquema de los niveles de riesgo asociados a distintos niveles de consumo; curva de alcoholemia tras beber; riesgo asociado a la conducción bajos distintos niveles de alcoholemia) y 2 dípticos para entregar al paciente: (uno informativo sobre los límites de riesgo para el consumo de alcohol en general y otro de autoayuda para modificar la conducta de riesgo: alcohol al volante). Consideramos que, además de incidir sobre el binomio alcohol-conducción, debíamos ofrecer un mensaje sobre los límites prudentes del consumo de alcohol a fin de que el paciente pudiera replantearse y modificar, si fuera necesario, no sólo su conducta vial, sino su consumo de alcohol, cuya reducción es el objetivo principal del estudio.

Aunque en la fase piloto no se preveía la distribución aleatoria en IB y IM, se procedió a la adscripción de pacientes a uno u otro método por semanas alternas a fin de probar la facilidad de este sistema de distribución, si bien se alteró esta distribución cuando convino a los objetivos del piloto (cada profesional debía haber administrado los dos tipos de intervención).

Se elaboraron cuestionarios para conocer la opinión para el personal participante acerca de la formación recibida, autoeficacia percibida y viabilidad estructural estimada del proyecto. Respondieron todas las personas que habían realizado intervenciones $(N=6)$,

\section{Estimación de la prevalencia y elaboración del perfil de los lesionados con presencia de alcohol en saliva}

Se registró el número total de pacientes asistidos en urgencias de traumatología por accidente de tráfico 
entre el 14 de Diciembre de 2000 y el 30 de Marzo de 2001, así como el número de pacientes cribados.

Se elaboró una ficha para recoger las variables de los lesionados en accidentes de tráfico (dia y hora de la asistencia, hora del accidente, edad, sexo, posición en vehículo, resultado de la saliva, resultado de la evaluación, intervención aplicada, derivación y seguimiento). Además de analizar la información contenida en las fichas abiertas, se recogió la misma información de todos los pacientes atendidos por accidente de tráfico durante el periodo consignado a fin de conocer las características de unos y otros y comprobar si la muestra estudiada podía considerarse representativa.

\section{Proceso y análisis de datos}

El estudio piloto se inició el 14 de Diciembre de 2000 y finalizó el 30 de Marzo de 2001.

Toda la información recogida de los pacientes, así como las encuestas de opinión del personal fueron procesadas informáticamente aplicándose técnicas de análisis estadístico descriptivo, fijando el nivel de sig- nificación estadística en valores $<0,5$ con la prueba de Fisher.

\section{RESULTADOS}

\section{Formación}

Por lo que respecta a la formación teórico-práctica de los profesionales (unas 10 horas), su resultado fue satisfactorio, a pesar de las reticencias iniciales y de las dificultades prácticas para su implementación. Los profesionales valoraron mayoritariamente la formación recibida, que consideraron útil $(80 \%)$ y adecuada a los objetivos $(80 \%)$. Se valoró especialmente el role-playing $(100 \%)$ y el material suministrado (80\%). El $80 \%$ consideró adecuado el tiempo dedicado a la formación teórica, mientras que el entrenamiento práctico sólo le pareció suficiente al $50 \%$ de participantes. Al final de la formación y antes de haber practicado la intervención, el $60 \%$ se consideraba suficientemente preparado para intervenir (ver Tabla 1).

\section{Tabla 1. ENCUESTA SOBRE LA FORMACIÓN RECIBIDA}

Respuestas según escala de Likert (0-4) agrupadas (1-3'), en porcentajes

\begin{tabular}{|c|c|c|c|}
\hline & 1 & 2 & 3 \\
\hline -El tiempo dedicado a las explicaciones teóricas es adecuado & & 20 & 80 \\
\hline -El tiempo dedicado al entrenamiento práctico es el adecuado & 20 & 30 & 50 \\
\hline - Los contenidos del seminario parecen adecuados a los objectivos & & 20 & 80 \\
\hline -El material entregado ha sido útil para conocer la materia & 10 & 10 & 80 \\
\hline -El role-playing permite descubrir nuevas herramientas de trabajo & & & 100 \\
\hline -El role-playing ayuda a entender la importancia de la comunicación & & 10 & 90 \\
\hline -En conjunto la formación ha sido útil & 10 & 10 & 80 \\
\hline $\begin{array}{l}\text {-Me considero ya preparado/da para hacer una intervención breve sobre alcohol en } \\
\text { un lesionado }\end{array}$ & 10 & 30 & 60 \\
\hline
\end{tabular}

$\mathrm{N}=10$ (contesta $100 \%$ )

11: absolutamente en desacuerdo o en desacuerdo; 2: no seguro; 3: de acuerdo o absolutamente de acuerdo.

Entre las observaciones, dicen desear más entrenamiento práctico las 3 personas que no han seguido la formación completa.

\section{Cribado}

Aunque el reactivo parecía sencillo de utilizar, cuando se llevaban explorados 97 pacientes, sólo se tenían 3 casos positivos (3,1\%). Esta soprendente escasez de salivas positivas obligó a revisar tanto el lote utilizado como la aplicación del método. El análisis del lote indicó su correcto funcionamiento en condiciones de laboratorio. Por lo que respecta a su aplicación práctica, se pudo comprobar que una insuficiente salivación de la torunda podía generar falsos negativos y que la frecuente sequedad de boca y cambios en la viscosidad de la saliva en accidentados dificultaba considerable- mente la utilización del método, incluso cuando el personal estaba perfectamente capacitado para emplearlo.

Es de señalar que, a partir de la revisión en profundidad del procedimiento y el correspondiente entrenamiento de los profesionales en su manejo, el porcentaje de casos con saliva positiva fue aumentando a medida que avanzaba el estudio. Ver figuras 2 y 3.

En la muestra total analizada (261 pacientes), aparecieron 28 salivas positivas: $\mathbf{1 0 , 7 \%}$.

En los primeros 97 pacientes estudiados hubo sólo 3 casos aislados con saliva positiva. 
Figura 3. Evolución del porcentaje de casos con saliva positiva ( $N=261$ )

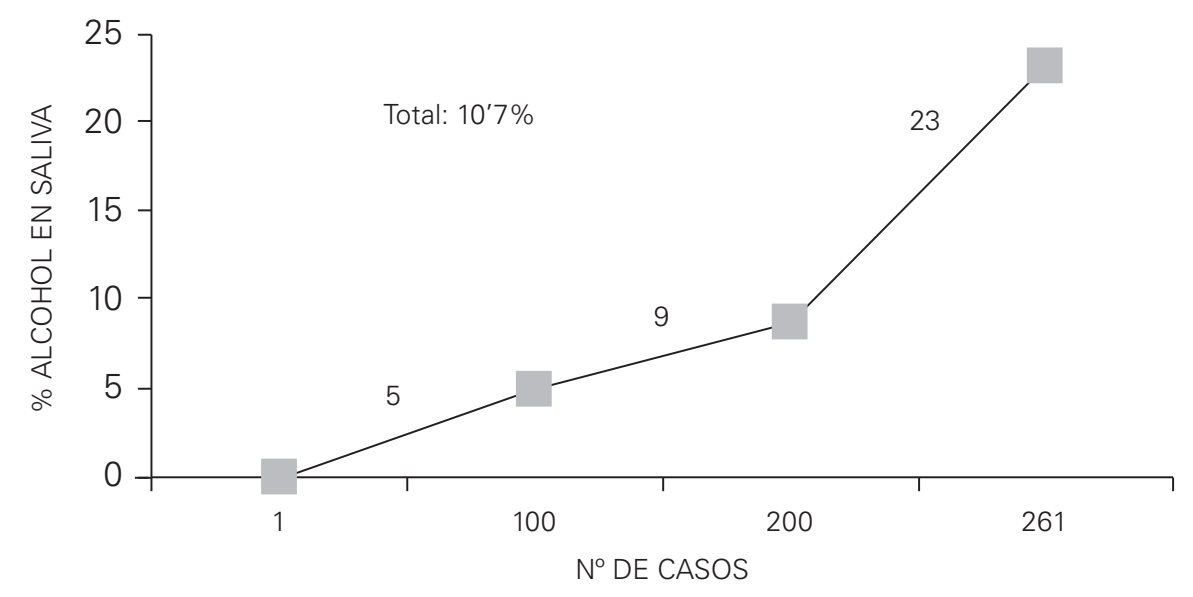

Figura 3. Evolución del porcentaje de casos con saliva positiva $(\mathrm{N}=\mathbf{2 6 1})$

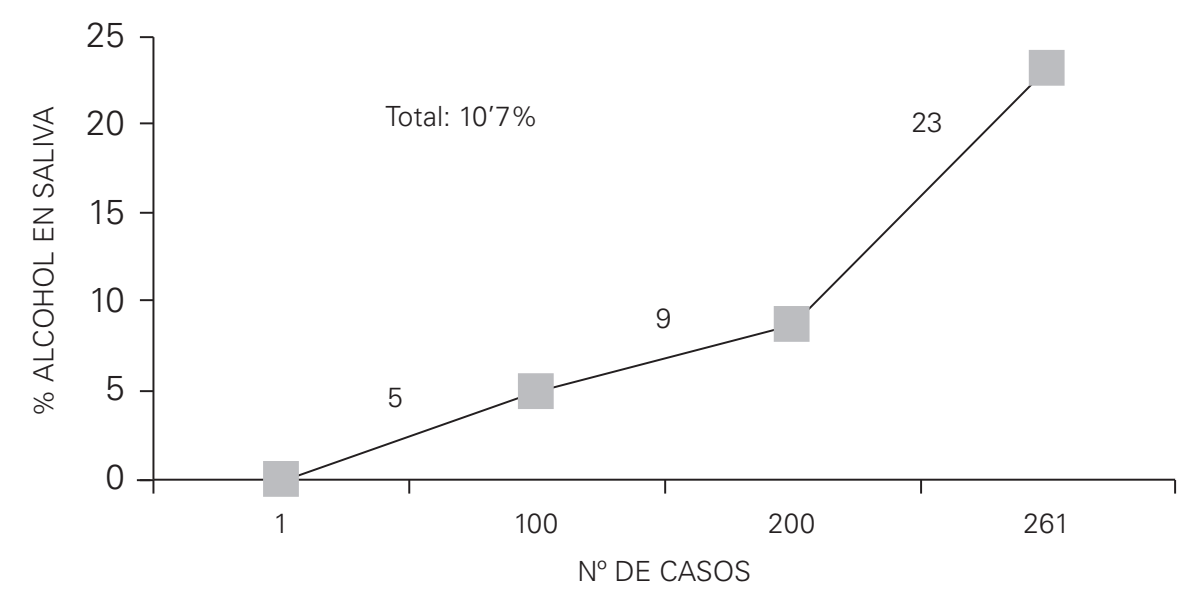

A partir del caso 98, y coincidiendo con la revisión crítica del procedimiento, empezaron a aparecer positivos $(5 \%$ en los 100 primeros casos, que ascienden al $9 \%$ entre el caso 101 y 200 y al $23 \%$ en el último tramo del estudio -casos 201 a 261-).

Si establecemos como punto de corte el momento en que se revisaron el reactivo y su procedimiento de aplicación, comprobamos la siguiente evolución:

Casos 1-97 (14 Diciembre a 20 de Enero): 3 casos positivos $(3,1 \%)$

Casos 98 a 261 (21 de Enero a 30 de Marzo): 25 casos positivos $(15,3 \%)$.

Esta evolución no se justificaba por ningún cambio en los criterios clínicos de selección.

La opinión de enfermería, escéptica con respecto al rendimiento de la prueba, fue sin embargo positiva con respecto a la viabilidad del cribado (ver Tabla 2).

La opinión predominante era que "el procedimiento no es complicado" (78,9\%), que "no existen dema- siados problemas para hacerlo" (55,0\%), que no "tengo escrúpulos deontológicos" para realizarlo $(55,0 \%)$ y que "en general, el paciente lo acepta bien" $(52,6 \%)$. El 52,2\% opinaba que "no hay bastante apoyo" del entorno a la hora de realizar el cribado.

Más divididas estuvieron las opiniones relativas al resto de aseveraciones, muy relacionadas con la presión asistencial en un servicio de urgencias. Sin embargo, la mayor parte de encuestados no estaba de acuerdo con que "no me acuerdo de pedirlo" (45,0\% del total: $64,3 \%$ porcentaje válido, teniendo en cuenta que un $30 \%$ no responde a esta pregunta).

Aunque todo el personal de enfermería fue instruido en el manejo del reactivo, sólo 10 personas habían sido formadas para realizar la intervención en los pacientes positivos.

Al comparar las respuestas del personal formado y no formado (Tabla 3), se observa una tendencia a conceder mayor importancia a la prueba por parte del personal 
Tabla 2. OPINIÓN DE LOS PROFESIONALES DEL SERVICIO SOBRE LAS DIFICULTADES PLANTEADAS PARA LA DETERMINACIÓN DEL ALCOHOL EN SALIVA (en porcentajes)

Respuestas según escala de Likert (0-4), agrupadas (1-31)

\begin{tabular}{|c|c|c|c|c|}
\hline & 1 & 2 & 3 & NC \\
\hline No existen demasiados problemas para hacerlo & 35 & 10 & 55 & \\
\hline Tengo escrúpulos deontológicos & 55 & 10 & 30 & 5 \\
\hline No hay tiempo para hacerlo & 30 & 15 & 35 & 20 \\
\hline Hay cosas más importantes en las que pensar & 35 & 10 & 40 & 15 \\
\hline No me acuerdo de pedirlo & 45 & 5 & 20 & 30 \\
\hline Distorsiona la dinámica de trabajo & 48 & 5 & 37 & 10 \\
\hline No hay bastante apoyo & 26 & 10 & 53 & 10 \\
\hline El procedimiento es complicado & 79 & & 10 & 10 \\
\hline En general, el paciente lo acepta bien & 21 & 21 & 53 & 5 \\
\hline
\end{tabular}

$\mathrm{N}=20 .{ }^{1} 1$ : absolutamente en desacuerdo o en desacuerdo; 2: no estoy seguro; 3: de acuerdo o absolutamente de acuerdo

Tabla 3. OPINIÓN DE LOS PROFESIONALES DEL SERVICIO SOBRE LAS DIFICULTADES PLANTEADAS PARA LA DETERMINACIÓN DEL ALCOHOL EN SALIVA (Comparación entre personal formado y no formado: porcentajes válidos)

\begin{tabular}{|c|c|c|c|c|}
\hline & Calif. & Formados & No formados & Signific. Chi C.Fisher \\
\hline 1. No existen demasiados problemas para hacerlo & $\begin{array}{l}1 \\
2 \\
3 \\
\end{array}$ & $\begin{array}{l}29 \\
14 \\
57 \\
\end{array}$ & $\begin{array}{c}38 \\
8 \\
54 \\
\end{array}$ & NS \\
\hline 2. Tengo escrúpulos deontológicos & $\begin{array}{l}1 \\
2 \\
3 \\
\end{array}$ & $\begin{array}{l}57 \\
43 \\
\end{array}$ & $\begin{array}{l}54 \\
17 \\
25 \\
\end{array}$ & NS \\
\hline 3. No hay tiempo para hacerlo & $\begin{array}{l}1 \\
2 \\
3 \\
\end{array}$ & $\begin{array}{l}33 \\
33 \\
33 \\
\end{array}$ & $\begin{array}{l}40 \\
10 \\
50 \\
\end{array}$ & NS \\
\hline 4. Hay cosas más importantes en las que pensar & $\begin{array}{l}1 \\
2 \\
3 \\
\end{array}$ & $\begin{array}{l}83 \\
17 \\
\end{array}$ & $\begin{array}{l}18 \\
18 \\
64 \\
\end{array}$ & 037 \\
\hline 5. No me acuerdo de pedirlo & $\begin{array}{l}1 \\
2 \\
3 \\
\end{array}$ & $\begin{array}{l}83 \\
17 \\
\end{array}$ & $\begin{array}{l}50 \\
12 \\
37 \\
\end{array}$ & NS \\
\hline 6. Distorsiona la dinámica de trabajo & $\begin{array}{l}1 \\
2 \\
3\end{array}$ & $\begin{array}{l}67 \\
33\end{array}$ & $\begin{array}{c}45 \\
9 \\
4 \\
\end{array}$ & NS \\
\hline 7. No hay bastante apoyo & $\begin{array}{l}1 \\
2 \\
3\end{array}$ & $\begin{array}{l}50 \\
50\end{array}$ & $\begin{array}{l}18 \\
18 \\
64\end{array}$ & NS \\
\hline 8. El procedimiento es complicado & $\begin{array}{l}1 \\
2 \\
3 \\
\end{array}$ & $\begin{array}{l}67 \\
29\end{array}$ & 100 & NS \\
\hline 9. En general, el paciente lo acepta bien & $\begin{array}{l}1 \\
2 \\
3\end{array}$ & $\begin{array}{l}29 \\
29 \\
43\end{array}$ & $\begin{array}{l}25 \\
17 \\
58\end{array}$ & NS \\
\hline
\end{tabular}

N: 20 (Personal formado': 7; Personal no formado 13). 1: absolutamente en desacuerdo o en desacuerdo; 2: no seguro; 3: de acuerdo o absolutamente de acuerdo.'Aunque se formó a 10 personas para la intervención, no todas participaban en el cribado de saliva.

entrenado, aunque la única diferencia que alcanza significación estadística se da en el ítem "hay cosas más importantes en las que pensar"afirmación con la que está de acuerdo el 63,6\% del personal no formado frente al $16,7 \%$ de las personas formadas (porcentajes válidos; significación de la Chi cuadrada de Fisher 0,037). 


\section{Intervención}

\section{Evaluación}

De los 261 pacientes cribados, 28 (10,7\%) tenía saliva positiva. De éstos, 18 (64,3\%) fueron evaluados según el protocolo, aunque hubo evaluaciones incompletas $(16,7 \%$ de los pacientes evaluados). Tanto los casos incompletos como los no evaluados se atribuyeron a causas diversas (falta de tiempo, alta adelantada...). Sólo en 1 caso $(3,6 \%)$ se debió a rechazo del paciente.

El AUDIT fue el único test cumplimentado en todos los casos evaluados, quedando catalogados como bebedores de riesgo el $36,8 \%$ de pacientes (15,8\% con 21 puntos: sospecha de dependencia).

Inventario de actitudes, conocimientos y conductas: aunque se aplicó en el $84,2 \%$ de casos, se completó de forma irregular (al no ser mesurable, no se puso tanto empeño en contestar todas sus preguntas).

Entre los datos recogidos con este instrumento contaba la prevalencia de accidentes de tráfico anteriores (con o sin víctimas). Es de destacar que el $72,2 \%$ de los lesionados con saliva positiva evaluados habían tenido ya al menos otro accidente de tráfico. El $50 \%$ declaraba no tener ni idea de su nivel de alcoholemia en el momento del accidente, el 22,2\% creía tener 0,5 y sólo 1 paciente consideró que podía tener $1 \mathrm{~g} / \mathrm{l}$.

El 38,9\% había sufrido alguna fractura de huesos anteriormente.

En cuanto a la escala de atribución al alcohol del accidente (escala 1-7), el $40 \%$ de lesionados que lo cumplimentaron admitía alguna relación ("algo" el 26,7\%; "bastante" o "totalmente": el 13,3\%).
En la regleta del cambio (escala 1-10), los pacientes se concentraron a la derecha de la "duda" y se posicionaron como "preparados para el cambio": el $62,3 \%$ puntúan 7 o más puntos y el $50 \%$ se concentra en la franja 9-10.

La Fig. 4 representa el proceso seguido por la muestra de pacientes estudiados.

El estudio piloto no preveía un análisis pormenorizado de la evaluación, que tampoco tendría sentido dada la pequeña cantidad de pacientes evaluados.

\section{Consejo}

El $57,1 \%$ de los lesionados con saliva positiva (el $88,9 \%$ de todos los pacientes evaluados), recibieron una intervención de consejo (50\% IM o 50\% IB).

La intervención fue en conjunto bien acogida por el paciente y las dificultades encontradas derivaron más de las circunstancias (exceso de trabajo, errores de interpretación, pérdida / alta anticipada) que de la actitud del lesionado. Sólo un caso fue derivado, a petición del propio paciente.

Si bien la supervisión directa de las intervenciones no resultaba viable, se procedió a revisar la experiencia a posteriori, tanto a nivel individual como de grupo.

Al final de la experiencia, se pasó una encuesta de opinión a los profesionales que habían hecho la intervención (Tabla 4).

El 66,7\% del personal que realizó las intervenciones (6 personas de las 10 formadas debido a incompatibilidad con otras tareas en las 4 restantes) se había sentido cómodo y capacitado para la tarea y creía que la intervención serviría para algo. Sin embargo, también el $66,7 \%$ consideraba que el estudio piloto había representado una sobrecarga de trabajo y sólo el 16,7\% consideraba que su tarea había sido valorada por sus compañeros. A la hora de opinar sobre la implementa-

Figura 3. Evolución del porcentaje de casos con saliva positiva $(N=261)$

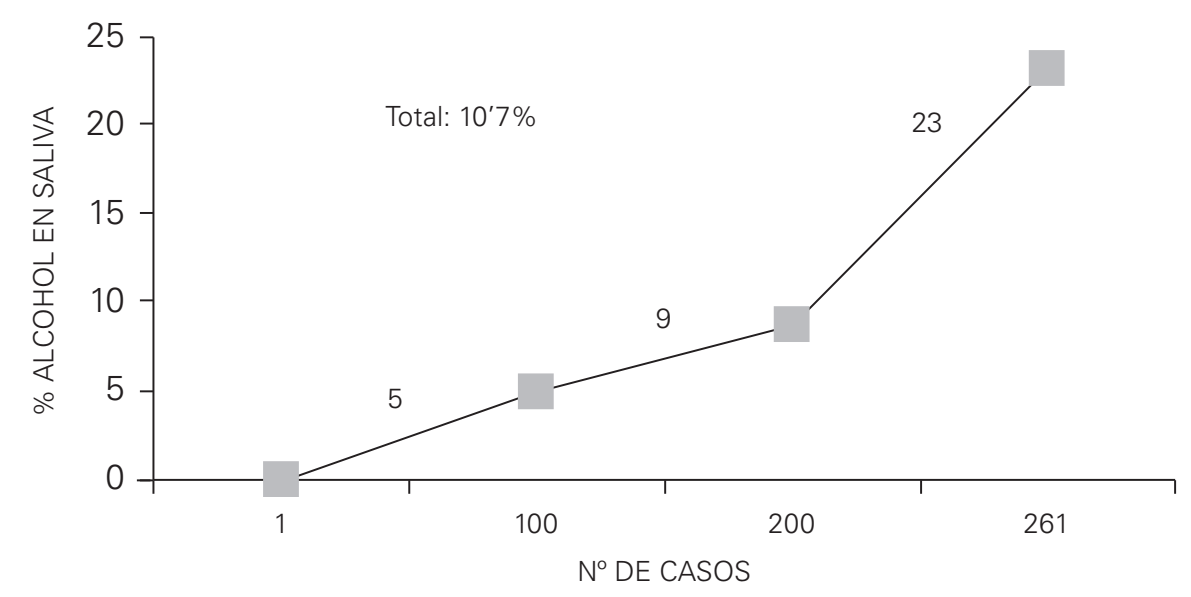


Tabla 4. Evaluación de la experiencia por parte del personal que ha participado en el estudio piloto. Cuestionario de opinión

Respuestas según escala de Likert (0-4) agrupadas (0-3), en porcentajes.

\begin{tabular}{|c|c|c|c|}
\hline & 1 & 2 & 3 \\
\hline ¿Consideras que este programa piloto ha representado una sobrecarga de trabajo para ti? & 17 & 17 & 67 \\
\hline ¿Tienes la impresión de que la intervención realizada servirá para algo? & 17 & 17 & 67 \\
\hline ¿Te has sentido capacitado/a y cómodo/a al hacer la intervención? & & 42 & 67 \\
\hline $\begin{array}{l}\text { ¿Crees que tus compañeros de trabajo han valorado tu tarea? } \\
\text { ¿Consideras que este programa piloto podría ser implementado en la práctica habitual del }\end{array}$ & 33 & 50 & 17 \\
\hline servicio de urgencias? & 33 & 33 & 33 \\
\hline
\end{tabular}

$\mathrm{N}=6$ (contesta 100\%)

1: absolutamente en desacuerdo o en desacuerdo; 2: no seguro; 3: de acuerdo o absolutamente de acuerdo. Pregunta abierta: ¿Qué cambiarías para un mejor rendimiento del programa? 2 personas $(33,3 \%)$ la contestan y aluden al método de cribado.

ción de este programa en la práctica habitual del servicio de urgencias, las respuestas se dividían a partes iguales $(33,3 \%)$ entre quienes lo consideraban o no viable y quienes no estaban seguros de ello.

Es de señalar que, a la pregunta abierta sobre si cambiarían algo para mejorar el rendimiento de programa, el 33,3\% sugería mejorar el sistema de cribado.

Estimación de la prevalencia y perfil de los lesionados en accidente de tráfico con presencia de alcohol en saliva

Entre el 14 de Diciembre de 2000 y el 30 de Marzo de 2001 se incorporaron al estudio 264 lesionados que reunían criterios de inclusión (accidentados de tráfico de 18 o más años). Se aplicó el test del alcohol en saliva a 261 de ellos, con resultado positivo en 28 pacientes $(10,7 \%)$.

\section{Perfil de la muestra total}

La muestra está constituida por 264 pacientes (Tabla 5), con una edad media de 29 años, un predominio de varones (69\%) y de conductores (67\%). Acu- dieron de preferencia en domingo o sábado (19\%, respectivamente) y en horario diurno -08.00 a 20.00(60\%). El tiempo transcurrido entre el accidente y la hora de asistencia había sido de 1 h, 33' de media (mediana: 1 hora). Ingresó el 14\%.

La población total de pacientes atendidos por accidente de tráfico durante el mismo periodo en el Hospital de Traumatología y Rehabilitación ( $\mathrm{N}=1544)$ tenía 34 años de edad media, un 51\% de conductores, un $61,2 \%$ de varones y un $11 \%$ de ingresos.

De los 264 pacientes elegibles, $3(1,1 \%)$ rechazaron la prueba de saliva: 2 varones (25 y 45 años) conductores de coche y una mujer (26 años) pasajera.

De los 261 que entraron en el estudio de la saliva, $28(10,7 \%)$ presentaron alcohol en saliva y $89,3 \%$ fueron negativos.

\section{Perfil de la submuestra de lesionados con saliva positiva}

Se trataba de lesionados con una media de edad de 28,4 , varones $(75 \%)$, conductores $(71,4 \%)$, atendidos

\begin{tabular}{|c|c|c|c|c|c|c|c|}
\hline \multicolumn{8}{|c|}{ Tabla 5. Perfil de los pacientes estudiados ( $N=264$, porcentajes) } \\
\hline $\begin{array}{l}\text { Edad } \\
\text { media' }\end{array}$ & Sexo & Posición & $\begin{array}{c}\text { Día } \\
\text { semana }\end{array}$ & $\begin{array}{c}\text { Hora } \\
\text { asistencia }\end{array}$ & $\begin{array}{c}\text { Tiempo transcurrido } \\
\text { desde accidente (horas) }\end{array}$ & Ingresa & Saliva \\
\hline \multirow[t]{3}{*}{$\begin{array}{l}29 \\
\text { (DS 11) }\end{array}$} & H 69 & $\begin{array}{l}\text { Conductores } \\
\text { CC } 34 \\
\text { CM } 26 \\
\text { CCM } 6\end{array}$ & $\begin{array}{l}\text { Do } 19 \\
\text { L } 11 \\
\text { Ma } 13 \\
\text { Mi } 12\end{array}$ & D 60 & $\begin{array}{c}1,33 \mathrm{~h} \\
\text { (media) }\end{array}$ & S 14 & \multirow{3}{*}{$\begin{array}{l}\text { P11 } \\
\text { N } 88 \\
\text { R } 1\end{array}$} \\
\hline & \multirow[t]{2}{*}{ M 31} & Total 67 & $\begin{array}{l}\text { J } 13 \\
\text { V } 13\end{array}$ & N 40 & \multirow{2}{*}{$\begin{array}{c}1 \mathrm{~h} \\
\text { (mediana) }\end{array}$} & \multirow[t]{2}{*}{ N 86} & \\
\hline & & $\begin{array}{l}\text { Pasajeros } 28 \\
\text { Peatones } 12\end{array}$ & S 19 & & & & \\
\hline $\begin{array}{l}\text { 1 El cribad } \\
\text { CC: condu } \\
\text { L: lunes; } \\
\text { D: 08-20; }\end{array}$ & $\begin{array}{l}\text { uía, pol } \\
\text { coche; } \\
\text { artes; } \\
08 \quad S: \\
\end{array}$ & $\begin{array}{l}\text { tocolo, a los me } \\
\text { conductor moto; } \\
\text { iércoles; J: jueve } \\
\text { :no. P: positivo; }\end{array}$ & $\begin{array}{l}\text { hores de } 18 \\
\text { CCM: cond } \\
\text { es; V: vierne: } \\
\text { N: negativo }\end{array}$ & $\begin{array}{l}\text { s. } \\
\text { r ciclomotor } \\
\text { sábado; Do: } \\
\text { rechazo }\end{array}$ & mingo & & \\
\hline
\end{tabular}


predominantemente en domingo $(42,9 \%)$ y sábado $(28,6 \%)$ e indistintamente de día o de noche $(53,6 \%$ versus 46,4\%). Quedaron ingresados un $25 \%$.

\section{Perfil comparado de las submuestras de lesiona- dos con saliva positiva y negativa}

Cuando se procede a la comparación de ambas submuestras (Tabla 6), se observa que las únicas diferencias significativas se refieren al día de la semana y a la necesidad de internamiento.

Los pacientes con saliva positiva acuden preferentemente en domingo (12 casos -42,9\%- frente a 38 $16,4 \%$ - de los negativos) y en sábado (8 casos -28,6\%frente a $41-17,7 \%$ - de los pacientes negativos), siendo la diferencia estadísticamente significativa ( $p 0,005)$. Quedan ingresados 7 (25\%) de los lesionados con presencia de alcohol en saliva y $27(11,8 \%)$ de los lesionados sin alcohol en saliva ( $p 0,05)$.

\section{DISCUSIÓN}

La identificación precoz, especialmente la identificación de un consumo problemático de alcohol concu- rrente con un accidente de tráfico, es el elemento de partida sobre el que asienta una intervención destinada al cambio de conducta (cuantía y circunstancias del consumo). El secreto de la IB parece radicar en su estilo motivacional. Motivar al paciente presupone motivar primero al profesional. De ahí que sea rentable invertir en la formación de los profesionales en un sentido holístico, trabajando asimismo su actitud y fomentando su compromiso. El personal ha de ser protagonista del proyecto, no su instrumento. Sin su motivación no hay estudio. Las barreras inicialmente detectadas (actitud escéptica de algunos profesionales), así como las anticipadas por los profesionales (resistencias del paciente, dificultades estructurales), fueron derribándose a medida que avanzaba el estudio.

El porcentaje de lesionados de tráfico a quienes se determinó la saliva $(17,1 \%)$ puede considerarse satisfactorio, si lo comparamos con otros estudios ${ }^{6,33}$, sobre todo teniendo en cuenta que no se pretendía hacer una cobertura universal, sino probar la viabilidad del diseño. Quedaban excluidos por protocolo, los menores de 18 años. La embriaguez y la condición de politraumatismo grave no eran excluyentes para el cribado, aunque la intervención -caso de resultado positivo- debía posponerse hasta que el paciente se hallara desintoxicado y en condiciones de responder.

Tabla 6. Perfil comparado entre los pacientes con saliva positiva y los pacientes con saliva negativa

\begin{tabular}{|c|c|c|c|}
\hline Total muestra $(\mathrm{N}=260)$ & Positivos (N= 28) & Negativos ( $\mathrm{N}=\mathbf{2 3 2}$ ) & Sig.Chi C-Fisher \\
\hline Edad media & 28 & 28 & NS \\
\hline \multicolumn{4}{|l|}{ Sexo } \\
\hline $\mathrm{H}$ & 75 & 68 & \multirow[t]{2}{*}{ NS } \\
\hline M & 25 & 32 & \\
\hline \multicolumn{4}{|l|}{ Posición } \\
\hline C & 71 & 66 & \multirow[t]{3}{*}{ NS } \\
\hline $\mathrm{Pa}$ & 21 & 29 & \\
\hline $\mathrm{Pe}$ & 7 & 4 & \\
\hline \multicolumn{4}{|l|}{ Día semana } \\
\hline Do & 43 & 16 & \multirow[t]{7}{*}{0,005} \\
\hline L & 4 & 12 & \\
\hline Ma & 11 & 14 & \\
\hline Mi & 4 & 13 & \\
\hline$J$ & 0 & 15 & \\
\hline V & 11 & 13 & \\
\hline S & 29 & 18 & \\
\hline \multicolumn{4}{|l|}{ Hora asistencia } \\
\hline D & 54 & 61 & \multirow[t]{2}{*}{ NS } \\
\hline $\mathrm{N}$ & 46 & 39 & \\
\hline \multicolumn{4}{|l|}{ Ingresa } \\
\hline$S$ & 25 & 12 & \multirow{2}{*}{0,05} \\
\hline N & 75 & 88 & \\
\hline
\end{tabular}

$\mathrm{H}$ : hombres; M: mujeres. CC: conductor coche; CM: conductor moto; CCM: conductor ciclomotor; Pa: Pasajero; Pe: peatón L: lunes; Ma: martes; Mi: miércoles; J:jueves; V:viernes; S:sábado; Do:domingo. D: 08-20; N: 20-08 S:sí; N:no 
La formación recibida se ha adecuado a las necesidades, destacando la satisfacción del personal formado, que se sentía, al finalizar las sesiones, capaz de realizar la intervención en el $60 \%$ de casos, porcentaje análogo al obtenido tras seminarios más extensos e intensivos de "formación de formadores"37.

La no-determinación en pacientes incluibles tuvo causas diversas, siendo la falta de tiempo la razón más invocada (el rechazo del paciente es mínimo: $1,1 \%)$. Por lo que respecta a las pérdidas de casos positivos, sólo 2 casos $(7,1 \%)$ se perdieron por rechazo del paciente (1 rechazó la evaluación y otro el consejo). En conjunto, el rechazo de los accidentados ha sido muy inferior al referido en otros trabajos ${ }^{38}$, aunque a la ausencia de seguimiento en la fase piloto puede haber favorecido la aceptación.

Es de destacar la creciente y decidida colaboración del personal de enfermería a medida que avanzaba el proyecto y en especial el compromiso de los profesionales formados, que se sintieron idóneos y protagonistas de la labor encomendada. La clara implicación, la flexibilidad en la oferta formativa y el apoyo continuado del equipo investigador contribuyeron a evitar la deserción, desmotivación y actitudes negativas señaladas en otros estudios. ${ }^{6,38}$

El porcentaje de lesionados con saliva positiva de nuestra muestra total $(10,7 \%)$ fue inferior al que habitualmente refiere la literatura (20-30\%) y al que aparece en la estadística de las alcoholemias realizadas en accidentados por la Guardia Urbana de Barcelona en el año 2000 (35,6\%). Sin embargo, el porcentaje de casos positivos fue ascendiendo a medida que progresaba el estudio, alcanzando la cota esperada (23\%) en el último mes (casos 201 a 261). Esta evolución podría explicarse por el entrenamiento exhaustivo en el manejo del test -que se incorporó al comprobarse las dificultades en la aplicación del mismo-, y por la experiencia adquirida, dado que no se modificaron los criterios de inclusión. En cualquier caso, persistió el problema de recogida de la muestra en pacientes con boca seca, que es referido ${ }^{17}$ como causa de resultados no válidos por la literatura internacional sobre el tema. De los métodos disponibles en el mercado, los más rentables parecen ser el test Q.E.D. y el ALCO-Screen, ambos basados en el mismo tipo de reacción enzimática que el Alcohol On-Site, también sensibles a cambios en la cantidad o viscosidad de la saliva y no exentos de inconvenientes.

Conclusiones: 1) La formación del personal es factible y útil. 2) El procedimiento es estructuralmente correcto. 3) El cribado es posible en urgencias, aunque las opiniones difieren en cuanto a la disponibilidad de tiempo.4) El paciente lo acepta bien. 5) El test de saliva es fiaable, siempre y cuando se recoja muestra suficiente. 6) El personal se siente capacitado y cómodo para hacer la intervención y tiene la impresión de que su consejo será útil para el paciente, aunque con- sidera que el programa representa una sobrecarga de trabajo y muestra una opinión dividida respecto a la posibilidad de implementarlo en la práctica habitual del servicio de urgencias. 7) Los lesionados con saliva positiva acuden más en fin de semana y son más hospitalizados.

Estas conclusiones comportan las siguientes implicaciones prácticas para la fase principal del estudio:

-Se presentará el resultado de la fase piloto a toda la plantilla del servicio de urgencias a fin de favorecer su motivación e implicación en el proyecto.

-Se mantendrá un programa de entrenamiento flexible (inclusive de la aplicación del reactivo en saliva y orina) con supervisión continuada y refuerzo positivo.

-Se seguirá utilizando el mismo test para la detección cualitativa del alcohol en saliva. Tras las dificultades habidas, las enfermeras saben identificar los resultados no válidos; el nuevo protocolo incluirá la determinación en orina en los casos de saliva insuficiente. El test Alcohol On-Site permite hacer la prueba en los dos especímenes.

-Se potenciará la detección en los fines de semana (desde las 20,00h del viernes a las 20,00h del domingo) y en los politraumáticos.

Algunos turnos de guardia se reforzarán con personal especialmente remunerado para realizar la intervención, a fin de aliviar la sobrecarga de trabajo.

\section{AGRADECIMIENTOS}

El estudio en que se enmarca este trabajo está parcialmente financiado por el Fondo de Investigaciones Sanitarias (exp.nº1/0903).

Agradecemos sinceramente la colaboración de todo el personal de enfermería del Servicio de Urgencias del Hospital de Traumatología y Rehabilitación y muy especialmente la esforzada participación de los profesionales que se formaron y aplicaron tanto el cribado como la evaluación-intervención: Raquel Benedit, Carlota Cruzate, Rosa Espelt, , Rosa Felix, $\mathbf{M}^{a}$ Jose Garcia Calvache, Carolina Gómez, Miguel Lopez, Conchita Peña, Carme Riu, Aurora Vilella.

\section{BIBLIOGRAFIA}

(1) Babor TF, Grant M (eds.) Programme on Substance Abuse. Project on identification and management of alcohol-related problems. Report on Phase II: a randomized clinical trial of brief intervention in primary health care. Geneva, World Health Organization, 1992. 
(2) IsraelY, Hollander O, Sánchez-Craig M, Booker S, Miller V et al.: Screening for problem drinking and counseling by the primary care physician-nurse team. Alcoholism: Clinical \& Experimental Research 1996; 20: 1443-1450.

(3) Richmond R, Heather N, Wodak A, Kehoe L, Webster I: Controlled evaluation of a general practice-based brief intervention for excessive drinking. Addiction 1995; 90: 119-132.

(4) Heather N: Brief Intervention Strategies. En Hester RK \& Miller WR (eds.): Handbook of Alcoholism Treatment Approaches. Effective Alternatives. Boston, London Allyn \& Bacon, 1995a (pp.: $105-122$ )

(5) Deehan A, Templeton L, Taylor, Drummond C, Starng J. Are practice nurses an unexplored resource in the identification and management of alcohol misuse? Results from a study of practice nurses in England and Wales in 1995. J Adv Nurs 1998; 28: 592-597.

(6) Peters J, Brooker Ch, Mc Cabe C, Short N.: Problems encountered with opportunistic screening for alcoholrelated problems in patients attending an Accident and Emergency Department. Addiction 1998; 93 (4): 589594.

(7) Mclntosh MC, Leigh G, Baldwin NJ, Marmulak J. Reducing alcohol consumption. Comparing three brief methods in family practice. Can Fam Physician 1997; 43 : 1959-1962, 1965-1967.

(8) Ockene JK, Adams, A, Hurley TG, Wheeler EV, Hebert JR. Brief physician- and nurse practitioner- deliverd cunseling for high-risk drinkers: does it work? Arch Int Med 1999; 159: 2198-2205.

(9) Aalto M; Pekuri P; Seppä K: Primary health care nurses' and physicians' attitudes, knowledge and beliefs regarding brief intervention for heavy drinkers. Addiction 2001; 96: 305-311.

(10) Ockene JK; Wheeler EV; Adams A, Hurley TG; Hebert J.: Provider training for patient-centered alcohol counseling in a primary care setting. Arch Inter Med. 1997; 157: 2334-2341.

(11) Kahan M, Wilson L, Becker L: Effectiveness of physician-based interventions with problem drinkers: a review. Can Med Assoc J 1995; 152: 851-859.

(12) Degutis, LC; Schriver, JA.: Emergency Department (ED) physician screening for potential alcohol related problems in patients with minor injuries: results from an urban ED. 4thWorld Conference Amsterdam on Injury Prevention and Control. RAI-Amsterdam17-20 May 1998.

(13) Shepherd, D; Hargarten S.: Alcohol screening in the emergency department: blood versus saliva study (abstract). Ann. Emerg Med. 1994; 23:612.

(14) Clifford PR, Sparadeo F, Minugh PA, Nirenberg TD, Woolard $\mathrm{R}$, Longabaugh $\mathrm{R}$, Becker $\mathrm{B}$ : Identification of hazardous /harmful drinking among subcretically injured patients. Acad Emerg Med 1996; 3(3): 239-245.

(15) Tu GC, Kapur B, Israel Y: Characteristics of new urine, serum and saliva alcohol reagent strip. Alc Clin Exp Res 1992; 16 (2): 222-227.
(16) Jones AW: Measuring ethanol in saliva wih the QED enzymatic test device: Comparison with blood- and breath- alcohol concentrations. J Anal Toxicol 1995;19: 169-174.

(17) Bates ME, Martin CS: Immediate quantitative estimation of blood alcohol concentrations from saliva. J Stud Alc. 1997; 58 (5): 531-538.

(18) Phair IC, Mardel S, Bodiwala GG: Blood alcohol concentration measurement using a salivary reagent stick: a reliable tool for emergency departments? Arch Emerg Med 1990;7 (2): 69-72.

(19) Pate LA, Hamilton JD, Park RS, Strobel RM: Evaluation of a saliva alcohol test stick as a therapeutic adjunt in an alcoholism treatment program. J Stud Alc 1993; 54 (5): 520-521.

(20) Schwartz RH, Clark HW, Meek PS: Laboratory tests for rapid screening of drugs of abuse in the workplace: a review. J Addict Dis 1993; 12 (2): 43-56.

(21) Bien, Th.; Miller, W.R.; Tonigan, J.S.: Brief interventions for alcohol problems: a review. Addiction 1993; 88: 315336.

(22) Wilk Al; Jensen NM; Havighurst TC: Meta-analysis of randomized control trials addressing brief interventions in heavy alcohol drinkers. J Gen Int Med 1997; 12: 274283.

(23) Chafetz ME, Blane HT, Abram HS, Golner J, Lacey E et al.: Establishing treatment relations with alcoholics. J Nerv Ment Dis 1962; 134: 395-409.

(24) Chafetz ME, Blane HT, Abram HS, Clark E, Golner JH et al. Establishing treatment relations with alcoholics: a supplementary report. J Nerv Ment Dis 1964; 138: 390-393.

(25) D'Onofrio G, Bernstein E, Bernstein J, Woolard R, Brewer Ph A, Craig SA et al.: Patients with alcohol problems in the emergency department, part 2: Intervention and referral. Academic Emerg Med 1998; 5: 1210-1217.

(26) Bernstein E, Bernstein J, Levenson S: Project ASSERT: an ED based intervention to increase access to primary care, preventive services, and the substance abuse treatment system. Ann. Emerg Med 1997; 30: 181-189.

(27) Longabaugh R, Minugh PA, Nirenberg TD, Clifford PR, Becker B, Woolard R. Injury as a motivator to reduce drinking. Acad Emerg Med 1995; 2: 817-825.

(28) Gentinello LM, Duggan P, Drummond D et al.: Major injury as a unique opportunity to initiate treatment in the alcoholic. Am J Surg 1988; 156: 558-561.

(29) Antti-Poika I, Karaharju E, Roine R\& Salaspuro M: Intervention of heavy drinking: a prospective and controlled study of 438 consecutive injured male patients. Alcohol \&Alcoholism 1988; 23: 115-121.

(30) Gentinello, LM, Rivara FP, Donovan DM, Jurkovich GJ, Daranciang $E$ et al.: Alcohol Interventions in a trauma center as a means of reducing the risk of injury recurrence. Ponencia al 119th Meeting of the American Surgical Association, San Diego, 1999.

(31) Sommers MS, Dyehouse JM, Howe SR, Lemmink J.: Preventing alcohol-related motor vehicle injury with 
brief intervenction strategies: preliminary findings with 60 subjects. 42nd Annual Proceedings. Association for the Advancement of Automotive Medicine. October 57, 1998. Charlottesville, Virginia.

(32) Sommers MS, Dyehouse JM, Howe SR, Weeks A, Russell AC: Using brief interventions following alcohol-related motor vehicle crashes to reduce drinking and further injury. Ponencia presentada en ICADTS-2000, Estocolmo, 20-25 Mayo 2000.

(33) Jurkovich GJ; Rivara FP; Gurney, JG; Ries, R; Mueller, BA; Copass, M.: The effect of acute alcohol intoxication and chronic alcohol abuse on outcome from trauma. JAMA 1993; 270 (1): 51-56.

(34) Rivara, FP; Koepsell, TD; Jurkovich, GJ; Gurney, JG; Sodeberg, R.: The effects of alcohol abuse on readmission for trauma. JAMA 1993; 270 (16): 1962-1964.
(35) Rodríguez-Martos A: Medical education: the way ahead. WHO Ministerial Conference on Young People and Alcohol. Working Group 4: Health Systems and Alcohol. Stockholm, 19-21 February 2001.

(36) Alcohol On-Site. Assay for the Qualitative Detection of alcohol in urine and saliva. Roche Diagnostic Systems. Somerville, U.S. October 1996

(37) Colom J, Gual A. Un modelo de intervención integral sobre los problemas derivados del alcohol desde la atención primaria de salud: el programa Beveu Menys. JANO 2000 LIX: 93-96.

(38) Brooker Ch, Peters J, McCabe Ch, Short N: The views of nurses to the conduct of a randomised controlled trial of problem drinkers in an accident and emergency department. Int J Nursing Studies 1999; 36: 33-39.

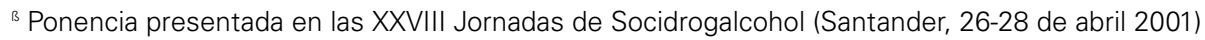


